\begin{tabular}{|c|c|}
\hline Title & Topology Optimization Based on Immune A Igorithm and Multigrid Method \\
\hline Author(s) & W atanabe, K.; Campelo, F.; Igarashi, H. \\
\hline Citation & $\begin{array}{l}\text { IEEE Transactions on Magnetics, 43(4), 1637-1640 } \\
\text { https://doi.org/10.1109/T MA G.2006.892259 }\end{array}$ \\
\hline Issue Date & 2007-04 \\
\hline Doc URL & http:/hdl.handle.net/2115/22087 \\
\hline Rights & $\begin{array}{l}\text { (C) } 2007 \text { IEEE. Personal use of this material is permitted. However, permission to reprint/Republish this material for } \\
\text { advertising or promotional purposes or for creating new collective works for resale or redistribution to servers or lists, } \\
\text { or to reuse any copyrighted component of this work in other works must be obtained from the IEEE. IEEE Transactions } \\
\text { on Magnetics } \\
\text {, vol. } 44 \text {, issue 4, 2007, pp. } 1637-1640\end{array}$ \\
\hline Type & article \\
\hline File Information & ITM43-4.pdf \\
\hline
\end{tabular}

Instructions for use 


\title{
Topology Optimization Based on Immune Algorithm and Multigrid Method
}

\author{
Kota Watanabe, F. Campelo, and H. Igarashi \\ Division of Systems Science and Informatics, Graduate School of Information Science and Technology, Hokkaido University, \\ Sapporo 060-0814, Japan
}

\begin{abstract}
This paper describes topology optimization of electromagnetic systems using the multigrid method. The topology optimization employs uniform rectangular finite elements, in which the multigrid method works effectively as a fast linear solver. Moreover, the present method uses a new optimization algorithm, based on the artificial immune systems paradigm, in which the multilevel search is carried out.
\end{abstract}

Index Terms-Finite-element method (FEM), immune algorithm, multigrid method, topology optimization.

\section{INTRODUCTION}

I $\mathrm{N}$ the shape optimization of electromagnetic systems based on field computation, the shape of the device being designed is usually expressed in terms of a small number of parameters. This leads to the problem of minimizing or maximizing an objective function of these parameters, which can effectively be solved using optimization methods, such as genetic algorithms, evolution strategies, or artificial immune systems.

In contrast, the topology-otimization (TO) approach seeks the optimal shape for a given device by making free modifications in the distribution of material within the search space, without the need of predefining any parameters (e.g., [1]). Moreover, the topological properties of the device can change during the optimization process. This paradigm is, therefore, able to deliver essentially new, unexpected results of good performance, because no prescribed shape and parameters are assumed.

Topology optimization, however, needs more complicated processes, and presents a larger number of degrees of freedom in comparison with traditional shape optimization. While intensive studies on this topic have been carried out in order to improve the optimization process, the computational cost of TO is still beyond the level required for practical uses even with modern computer performance. Moreover, conventional topology optimization algorithms sometimes yield too complicated shapes, which are not appropriate for practical applications.

Artificial imune systems (AIS) [4] are a family of computational methods drawing inspiration from theories about the mammalian immune system. AIS-based methods are becoming increasingly popular in a number of research fields, including parametric shape optimization, where a good balance between global and local exploration can be achieved at a reasonably low computational cost. Moreover, the fact that immune algorithms do not depend on any recombination operators (such as

Digital Object Identifier 10.1109/TMAG.2006.892259 the gentic algorithm's (GA's) crossover) can potentially make it simpler to implement.

These promising characteristics led to the development of an immune algorithm for topology optimization, which is presented in this work. The optimization technique, detailed in Section II, is coupled with the multigrid (MG) method for electromagnetic analysis (Section III). This method presents the characteristic of generating a hierarchy of meshes for a given problem, and is able to reduce the computational effort required for the evaluation of a given topology. It is accomplished by eliminating the higher-frequency residual components on a fine mesh, after a small number of iterations. The remaining residues are then projected onto a coarser mesh, in which they now have high frequency and can therefore be eliminated after another few iterative steps, and so on.

\section{TOPOLOGY OPtIMIZATION ALGORITHM}

In this work, an immune-based algorithm for topology optimization is used. This algorithm draws inspiration from the clonal selection principle (CSP) [4], and combines local and global search characteristics with repair operators in order to evolve complex distributions of material from random initial conditions. The algorithm also includes features for smoothing the topologies generated, in order to obtain feasible devices from an engineering point of view.

The proposed approach, called clonal selection algorithm for topology optimization (TopCSA), uses a simple 2-D binary matrix for representing the search space. The space is divided in a number of quadrilateral elements (corresponding to elements in a finite-element mesh) and each element is associated with a binary value indicating either the presence or the absence of material in that region of the space.

The algorithm starts by generating $N_{\text {pop }}$ initial distributions of material in the design space. Each distribution is called an antibody, according to our immunological metaphor, and can be generated either randomly or as a user-defined initial topology. The iterative cycle then starts, with the evaluation of the antibodies over an affinity function that measures their performance as solutions of the problem at hand. The antibodies are sorted 
according to their values of affinity, and the $N_{\text {sel }}$ best ones are selected for the clonal expansion: for a number of times given by

$$
N_{C}^{i}=\frac{\beta N_{\mathrm{pop}}}{i}
$$

where $i$ is the position of the antibody in the ranking, a copy (clone) of the antibody is generated, and is then subject to either the affinity maturation process or to one of the global operators, as explained below. After that, the clone is evaluated, and replaces the original antibody if it presents better performance.

The affinity maturation is an operator that targets the local improvement of the candidate solutions. It acts by introducing small local variations in the "surface" of the material (i.e., on elements that are set as filled and present at least one common interface with an empty element). One of these elements is randomly chosen, and then mutated according to the following procedure.

1) Determine the interface side of the element. If the element presents more than one interface side, select one randomly.

2) Randomly determine the mutation direction $( \pm 1)$.

3 ) If the direction is positive, the neighboring empty element is converted to filled; if it is negative, the filled element itself is converted to empty.

This operator presents some specific characteristics: First, it is a local operator, since it acts only on the immediate neighborhood of the affected elements. Second, the advance or retreat of the material boundary occurs only perpendicularly to the interface side.

There are three global operators that can affect a clone: cleaning, surface smoothing, and macromutation (see Fig. 1). These operators are both mutually exclusive and exclusive with the affinity maturation operator within a given generation of the algorithm.

The cleaning operator checks the neighborhood of all elements from the clone's distribution of material, and inverts the state of elements that present material boundaries at all four sides. In other words, it is responsible for removing floating pieces of material and small holes, which helps to smooth the inner parts of the material distribution.

The surface smoothing acts similarly to the cleaning operator, but instead of acting in elements completely surrounded by different ones, it operates on both sides of the material boundary, checking for elements that present three boundary faces (individual protruding filled elements or intruding empty elements).

Finally, the last of the global operators (macromutation) consists in randomly sorting a rectangular region in the clone's distribution of material, and setting all elements in the region to the same state, randomly chosen. This operator helps the algorithm explore new regions of the search space, to evolve shapes with holes in the middle of material regions, and to escape local optima.

After the clonal expansion cycle (cloning + selected operator), the antibodies that were not selected for cloning are replaced by new, randomly generated configurations of material, in a process here called diversity generation. The iterative cycle is repeated until some user-defined stop criterion is met.

Another strategy used for reducing the computational cost of the algorithm is the dynamic adaptation of the algorithm's parameters: as the optimization proceeds, the population is gradually reduced, and the diversity generation step is eventually turned off (in later generations, the chance of a random distribution of material to outperform an already evolved shape is negligibly small). At the same time, the $\beta$ parameter that regulates the number of clones generated around a given antibody increases, thus boosting the local evolution of the candidate solutions.

\section{A. Enforcing Smoothness}

In the topology optimization of engineering devices, there is a heavy concern about the smoothness of the solutions. Too rough, irregular, or perforated topologies may be too expensive or even unfeasible from an implementation point of view. Conversely, smoother, more homogeneous shapes are preferred, even if they lead to slightly worse performance of the device under consideration.

In order to allow the TopCSA to evolve smoother shapes, a simple strategy is employed: since the algorithm already possesses operators for smoothing the clones, we define an allowance $A_{S}$ for the affinity of clones subject to one of these operators. A smoothed clone is then allowed to replace the "parent" antibody if its affinity value is higher than $\left(1-A_{S}\right)$ times the affinity of the "parent." With this, it is possible to keep an evolutionary pressure in the direction of more regular-shaped topologies.

\section{MultiGRID MethoD}

It is known that the linear solvers, such as Gauss-Seidel methods, tend to eliminate the high-frequency components of the residue in linear equations given by the finite-element analysis more rapidly than the low-frequency components. The multigrid method is based on this property. The high-frequency residual components are eliminated on a fine mesh by small numbers of iterations of the linear solver. The remaining residual components are then projected onto a coarser mesh, in which they are now considered as high-frequency components that can again be eliminated by a small numbers of the iterations. The multigrid method solves given equations successively by performing this process. The geometric multigrid method, which is used in this work, requires the hierarchical meshes with different densities. In this study, the finer meshes are obtained by dividing each coarse element into four finer elements using the middle point of each edge. Although there are many variations in the multigrid method, all of these variations are based on the coarse grid correction. The procedure of the two-grid V-cycle method, which is the simplest multigrid method, is described. 


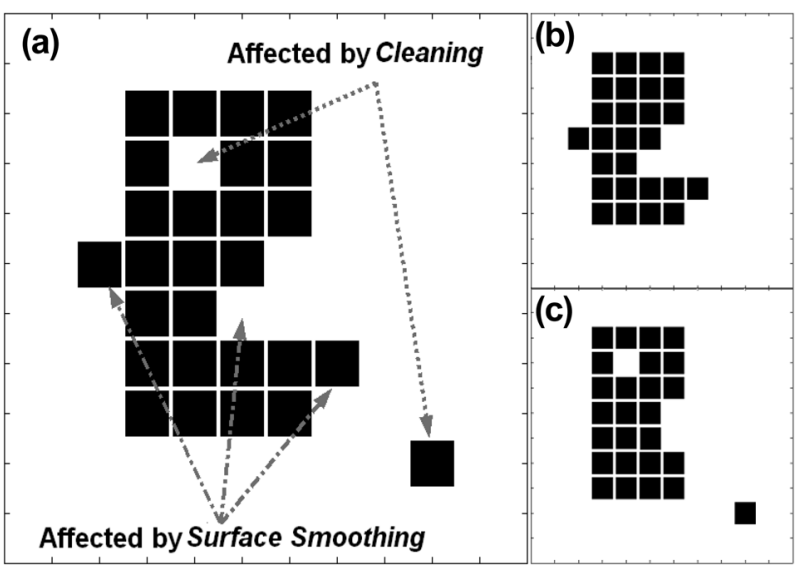

Fig. 1. Effect of two of the global operators. (a) Original distribution of material in a clone. (b) Resulting distribution after the cleaning operator. (c) Resulting distribution after surface smoothing.

$\underline{\text { Algorithm of multi-grid method. }}$

Step 1) A few iterations of the Gauss-Seidel method are applied to the given equation to obtain the a approximate solution $\{x\}$ (smoothing).

Step 2) The residual vector $\left\{r_{f}\right\}$ is calculated and projected onto a coarser mesh $\left\{r_{c}\right\}=[\mathrm{R}]\left\{r_{c}\right\}$, where $[\mathrm{R}]$ is the restriction matrix.

Step 3) The residual equation $\left[K_{c}\right]\left\{e_{c}\right\}=\left\{r_{c}\right\}$ is solved in order to obtain the error vector $\left\{e_{c}\right\}$.

Step 4) The error vector is projected onto the fine mesh $\left\{e_{f}\right\}=[\mathrm{R}]^{\mathrm{T}}\left\{e_{c}\right\}$.

Step 5) The solution is corrected $\{x\}^{\text {new }}=\{x\}+\left\{e_{f}\right\}$.

Step 6) The smoothing operation which is the same as Step 1) is applied to the system equation again.

Step 7) The convergence of the solution is tested. If the convergence condition is not satisfied, we go back to Step 2).

It is known that the performance of the multigrid method becomes worse for flat elements [2]. However, it works very effectively on the rectangular multilevel grid.

\section{RESULTS}

To show the performance of the present method, we analyze two electromagnetic systems. These analyses are performed on a personal computer with a Pentium 4 3.4-GHz CPU and 2-GB memory. The program code is written in $\mathrm{C}++$.

\section{A. Optimization of MRI Coil}

First, we analyze an optimization problem for the coil of magnetic resonance imaging (MRI) shown in Fig. 2 [3]. In this problem, the shape of the coil is optimized so that the magnetic field in the rectangular domain becomes uniform. The magnetic field is obtained by the finite-element (FE) analysis with the

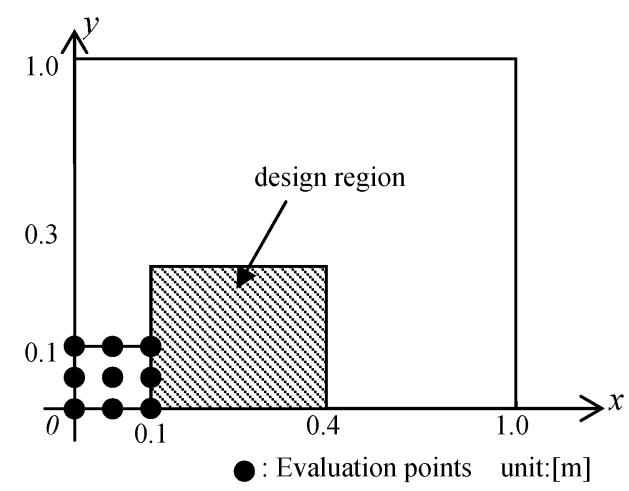

Fig. 2. Analysis region for MRI coil.

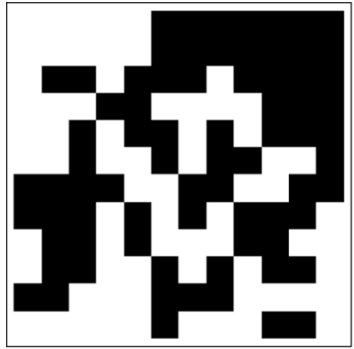

(a)

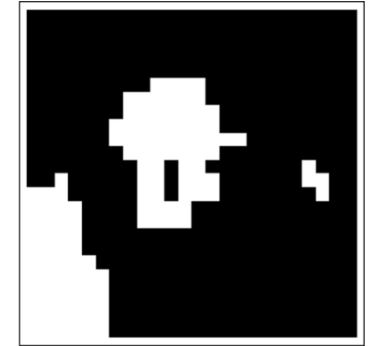

(b)

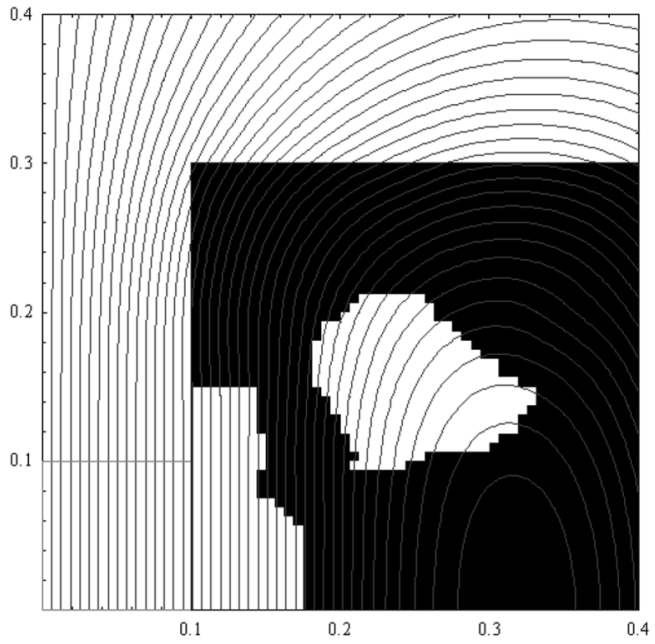

(c)

Fig. 3. Optimized shape of the coil (black region). (a) Initial distribution (12 $\times 12)$. (b) 275 th generation $(24 \times 24)$. (c) 1367 th generation $(48 \times 48)$.

multigrid method mentioned before. The objective function $f$ is defined as follows:

$$
f=\sum_{i=1}^{9}\left|B_{x}^{i}\right|+\sigma\left(B_{y}\right)
$$

where $B_{x}^{i}$ is the $x$ component of the magnetic flux density at the evaluation point $i$ shown in Fig. 2, and $\sigma\left(B_{y}\right)$ is the standard deviation of the $y$ component of the magnetic flux density.

Fig. 3 shows some results obtained when using the proposed method. The initial distribution of coil is set randomly and the resolution of design region is $12 \times 12$ [Fig. 3(a)]. In the process of optimization, the solution shows a tendency toward smoother 
TABLE I

Calculation Time Compared With the ICCG METHOD IN THE MRI COIL PROBLEM

\begin{tabular}{lcc}
\hline & $\begin{array}{c}\text { present method } \\
\text { (multi-grid) }\end{array}$ & $\begin{array}{r}\text { conventional } \\
\text { method (ICCG) }\end{array}$ \\
\hline CPU time [sec] & 4367 & 20965 \\
\hline
\end{tabular}

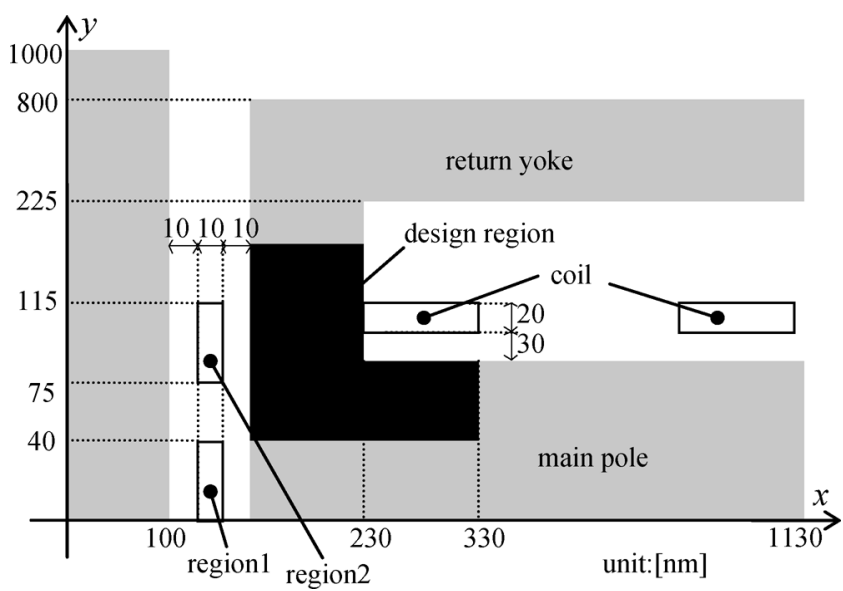

Fig. 4. Analysis region for magnetic head, current density in coil $0.1\left(\mathrm{~A} / \mathrm{m}^{2}\right)$, permeability of magnetic material $1000 \mu_{0}$.

shapes due to the global operators. Fig. 3(c) shows the optimized shape of coil after 10000 evaluations of the performance function and the contour lines of magnetic flux density. We can see that the present method evolved an optimal solution with a smooth shape, which is more appropriate for practical applications. Table I shows the calculation time using the multigrid method, compared with the incomplete Cholesky conjugate gradient (ICCG) method which is commonly used in FE analysis. We can see that the multigrid method is much faster than the conventional method.

\section{B. Optimization of Perpendicular Magnetic Recording Head}

Next, we analyze a perpendicular magnetic recording head shown in Fig. 4 [1]. The aim of this problem is to maximize the flux density in the region 1 , and to minimize the flux density in region 2 . The objective function is defined as the same in [1]

$$
f=w_{1} S_{1} \sum_{i=1}^{n_{1}} \frac{1}{\left(B_{x}^{i}\right)^{2} \Delta^{i}}+w_{2} \sum_{i=1}^{n_{2}} \frac{\left(B_{x}^{i}\right)^{2} \Delta^{i}}{S_{2}}
$$

where $S_{1}$ and $S_{2}$ are the areas of region 1 and region 2, respectively, and $\Delta^{i}$ is the area of the $i$ th element. $w_{1}$ and $w_{2}$ are the weighting coefficients, which are chosen as $w_{1}=1$ and $w_{2}=2 \times 10^{4}$. The resolution of the design region is fixed as $(37 \times 40)$ and the number of elements for FE analysis in

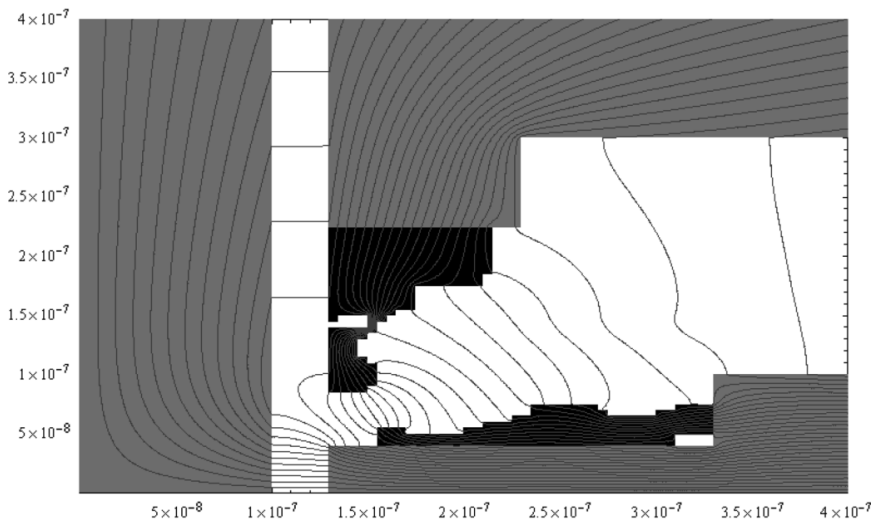

Fig. 5. Optimized shape and contour line of flux density.

TABLE II

Calculation Time Compared With the ICCG Method in the Magnetic Recording Head Problem

\begin{tabular}{lcc}
\hline & $\begin{array}{c}\text { present method } \\
\text { (multi-grid) }\end{array}$ & $\begin{array}{r}\text { conventional } \\
\text { method (ICCG) }\end{array}$ \\
\hline CPU time [hour] & 8.04 & 100.16 \\
\hline
\end{tabular}

the design region is $(148 \times 160)$. The total number of elements in the whole analysis region is 723200 and the number of unknowns is 724905 . The shape after 1000 evaluations is shown in Fig. 5. This figure shows that the present method yields a practical shape though the initial shape is given randomly.

Finally, to show the effect of the multigrid method, Table II shows the calculation time of this method compared with the ICCG. We can see that the multigrid method is much faster than the conventional method. It seems that the computational cost of the present method is compatible with the level required for practical uses with modern computer performance.

\section{REFERENCES}

[1] Y. Okamoto, M. Ohtake, and N. Takahashi, "Magnetic shield design of perpendicular magnetic recording head by using topology optimization technique," IEEE Trans. Magn., vol. 41, no. 5, pp. 1788-1791, May 2005.

[2] K. Watanabe, H. Igarashi, and T. Honma, "Convergence of multigrid method for edge-based finite-element method," IEEE Trans. Magn., vol. 39, no. 3, pt. 1, pp. 1674-1676, May 2003.

[3] C.-H. Im, H.-K. Jung, and Y.-J. Kim, "Hybrid genetic algorithm for electromagnetic topology optimization," IEEE Trans. Magn., vol. 39, no. 5, pt. 1, pp. 2163-2169, Sep. 2003.

[4] L. N. de Castro and J. Timmis, Artificial Immune Systems: A New Computational Intelligence Approach. Berlin, Germany: Springer-Verlag, 2002.

Manuscript received March 24, 2006 (e-mail: watanabe@ssi.ist.hokudai.ac. jp). 